\title{
A COLLOCATION METHOD FOR A HYPERSINGULAR BOUNDARY INTEGRAL EQUATION VIA TRIGONOMETRIC DIFFERENTIATION
}

\author{
RAINER KRESS
}

Communicated by Paul Martin

\begin{abstract}
Revisiting the author's paper from 1995 on this topic, a fully discrete collocation method is proposed for the hypersingular integral equation arising from the doublelayer approach for the solution of Neumann boundary value problems in two dimensions which is based on trigonometric differentiation to discretize the principal part of the hypersingular operator. Convergence in a Sobolev space setting is proven and the spectral convergence of the method is exhibited by numerical examples.
\end{abstract}

1. Introduction. The scattering of time-harmonic acoustic or electromagnetic waves from infinitely long cylindrical obstacles is modeled by exterior boundary value problems for the Helmholtz equation in two dimensions. In this paper we will be concerned with the Neumann boundary condition, i.e., scattering from sound-hard or nonconducting obstacles. Let $\Omega \subset \mathbf{R}^{2}$ be a simply connected bounded domain with infinitely differentiable boundary $\partial \Omega$ and unit normal vector $\nu$ directed into the exterior of $\Omega$. Given $g \in H^{-1 / 2}(\partial \Omega)$, the exterior Neumann problem for the Helmholtz equation consists of finding a solution $u \in H_{\mathrm{loc}}^{1}\left(\mathbf{R}^{2} \backslash \bar{\Omega}\right)$ to

$$
\Delta u+\kappa^{2} u=0 \quad \text { in } \mathbf{R}^{2} \backslash \bar{\Omega}
$$

with wave number $\kappa>0$ that satisfies

$$
\frac{\partial u}{\partial \nu}=g \quad \text { on } \partial \Omega
$$

Received by the editors on June 21, 2013, and in revised form on September 30, 2013. 
in the weak sense and the Sommerfeld radiation condition

$$
\lim _{r \rightarrow \infty} \sqrt{r}\left(\frac{\partial u}{\partial r}-i k u\right)=0, \quad r=|x|,
$$

uniformly for all directions. By

$$
\Phi(x, y)=\frac{i}{4} H_{0}^{(1)}(\kappa|x-y|), \quad x \neq y,
$$

we denote the fundamental solution to the two-dimensional Helmholtz equation in terms of the first kind Hankel function of order zero. Trying to find the solution in the form of a double-layer potential

$$
u(x)=\int_{\partial \Omega} \frac{\partial \Phi(x, y)}{\partial \nu(y)} \psi(y) d s(y), \quad x \in \mathbf{R}^{2} \backslash \bar{\Omega},
$$

with density $\psi \in H^{1 / 2}(\partial \Omega)$ leads to the hypersingular integral equation

$$
T \psi=g
$$

with the hypersingular operator $T: H^{1 / 2}(\partial \Omega) \rightarrow H^{-1 / 2}(\partial \Omega)$ given by

$$
(T \psi)(x):=\frac{\partial}{\partial \nu(x)} \int_{\partial \Omega} \frac{\partial \Phi(x, y)}{\partial \nu(y)} \psi(y) d s(y), \quad x \in \partial \Omega .
$$

Provided $-\kappa^{2}$ is not an eigenvalue for the Laplace operator in $\Omega$ with homogeneous Neumann condition on $\partial \Omega$, for each $g \in H^{-1 / 2}(\partial \Omega)$ the equation (1.2) has a unique solution $\psi \in H^{-1 / 2}(\partial \Omega)$ (for details in the three-dimensional case, see [8]).

The transformation

$$
\begin{aligned}
(T \psi)(x)= & \frac{\partial}{\partial s(x)} \int_{\partial \Omega} \Phi(x, y) \frac{\partial \psi}{\partial s}(y) d s(y) \\
& +\kappa^{2} \nu(x) \cdot \int_{\partial \Omega} \Phi(x, y) \nu(y) \psi(y) d s(y), \quad x \in \partial \Omega
\end{aligned}
$$

of the hypersingular operator $T$ is known as Maue's formula [12] and reflects the hypersingularity of $T$. Here, $\partial / \partial s$ denotes the derivative on $\partial \Omega$ with respect to arc length and the dot indicates the bilinear inner product between vectors in $\mathbf{R}^{2}$ or $\mathbf{C}^{2}$. For a proof of (1.3) for the limiting potential theoretic case $\kappa=0$, we refer to [11], and the proof for the three-dimensional Helmholtz case can be found in [7]. Maue's formula reduces the hypersingular operator acting on the density $\psi$ 
by a singular operator given in terms of a derivative of a single-layer potential acting on the derivative $\partial \psi / \partial s$ of the density. It suggests discretizing (1.2) via numerical differentiation of a discretization of the single-layer operator in order to take advantage of the simpler structure of the latter. Observing Atkinson's [1] remark:

... the most efficient numerical methods for solving boundary integral equations on smooth planar boundaries are those based on trigonometric polynomial approximations, and such methods are sometimes called spectral methods. When calculations using piecewise polynomial approximations are compared with those using trigonometric polynomial approximations, the latter are almost always the more efficient,...

in this paper we propose a collocation method for solving the hypersingular integral equation (1.2) via Maue's formula using trigonometric interpolation and differentiation.

To this end, we first need to parameterize the boundary integral equation (1.2). We assume that the boundary curve $\partial \Omega$ is described by a regular infinitely differentiable and $2 \pi$-periodic parametric representation of the form

$$
\partial \Omega=\{z(t): 0 \leq t \leq 2 \pi\}
$$

satisfying $\left|z^{\prime}(t)\right|>0$ for all $t$. After introducing the parameterized single-layer potential operator $S: H^{-1 / 2}[0,2 \pi] \rightarrow H^{1 / 2}[0,2 \pi]$ by

$$
(S \varphi)(t):=\frac{i}{4} \int_{0}^{2 \pi} H_{0}^{(1)}(\kappa|z(t)-z(\tau)|) \varphi(\tau) d \tau, \quad 0 \leq t \leq 2 \pi,
$$

and the differentiation operator $D: H^{1 / 2}[0,2 \pi] \rightarrow H^{-1 / 2}[0,2 \pi]$ by

$$
D \varphi:=\varphi^{\prime}
$$

the parameterized version of Maue's formula (1.3) reads

$$
\left|z^{\prime}\right|(T \psi) \circ z=D S D \varphi+\kappa^{2} z^{\prime} \cdot S\left(z^{\prime} \varphi\right)
$$

for $\varphi=\psi \circ z$. 
Due to the logarithmic behavior of the Hankel function $H_{0}^{(1)}(t)$ as $t \rightarrow 0$, the kernel

$$
H(t, \tau):=\frac{i}{4} H_{0}^{(1)}(\kappa|x(t)-x(\tau)|)
$$

of the operator $S$ has a logarithmic singularity. Therefore, following the presentation in [8], we split $H$ into

$$
H(t, \tau)=H_{1}(t, \tau) \ln \left(4 \sin ^{2} \frac{t-\tau}{2}\right)+H_{2}(t, \tau)
$$

where

$$
\begin{aligned}
& H_{1}(t, \tau):=-\frac{1}{4 \pi} J_{0}(\kappa|x(t)-x(\tau)|) \\
& H_{2}(t, \tau):=H(t, \tau)-H_{1}(t, \tau) \ln \left(4 \sin ^{2} \frac{t-\tau}{2}\right)
\end{aligned}
$$

and $J_{0}$ denotes the Bessel function of order zero. The kernels $H_{1}$ and $\mathrm{H}_{2}$ turn out to be infinitely differentiable. In particular, using the Taylor expansions for the Bessel and Neumann functions $J_{0}$ and $Y_{0}$ one can deduce the diagonal term

$$
H_{2}(t, t)=\frac{i}{4}-\frac{C}{2 \pi}-\frac{1}{2 \pi} \ln \left(\frac{\kappa}{2}\left|x^{\prime}(t)\right|\right), \quad 0 \leq t \leq 2 \pi,
$$

with Euler's constant $C$.

In order to write equation (1.2) in a form that exhibits the logarithmic singularities we introduce the following operators. We begin with the leading part of the single-layer operator $S$ given by

$$
\left(S_{0} \varphi\right)(t):=\frac{1}{2 \pi} \int_{0}^{2 \pi} \ln \left(4 \sin ^{2} \frac{t-\tau}{2}\right) \varphi(\tau) d \tau, \quad 0 \leq t \leq 2 \pi .
$$

Its basic properties follow from the fact that the trigonometric basis functions $f_{m}(t):=e^{i m t}$ for $m \in \mathbf{Z}$ are eigenfunctions, i.e.,

$$
S_{0} f_{m}=\beta_{m} f_{m}
$$

with the eigenvalues $\beta_{m}=-1 /|m|$ for $m \neq 0$ and $\beta_{0}=0$. This, in particular, implies that $S_{0}: H^{p}[0,2 \pi] \rightarrow H^{p+1}[0,2 \pi]$ is bounded for all $p \in \mathbf{R}$ (see [11, Theorem 8.22]). The principal part of the operator on 
the right-hand side of (1.5) can now be introduced as

$$
T_{0}:=D S_{0} D+M,
$$

where $M$ is the mean value operator given by

$$
M: g \longmapsto \frac{1}{2 \pi} \int_{0}^{2 \pi} g(t) d t .
$$

From

$$
T_{0} f_{m}=\gamma_{m} f_{m}
$$

for $m \in \mathbf{Z}$ with $\gamma_{m}=|m|$ for $m \neq 0$ and $\gamma_{0}=1$, we observe that $T_{0}: H^{p}[0,2 \pi] \rightarrow H^{p-1}[0,2 \pi]$ is an isomorphism.

Now we can write (1.2) in the form

$$
T_{0} \varphi-D A D \varphi-B \varphi=f
$$

where $f=-2\left|z^{\prime}\right|^{-1} g \circ z$ and $A$ and $B$ are integral operators of the form

$$
\begin{aligned}
& (A \varphi)(t):=\frac{1}{2 \pi} \int_{0}^{2 \pi}\left\{K_{A}(t, \tau) \ln \left(4 \sin ^{2} \frac{t-\tau}{2}\right)+L_{A}(t, \tau)\right\} \varphi(\tau) d \tau \\
& (B \varphi)(t):=\frac{1}{2 \pi} \int_{0}^{2 \pi}\left\{K_{B}(t, \tau) \ln \left(4 \sin ^{2} \frac{t-\tau}{2}\right)+L_{B}(t . \tau)\right\} \varphi(\tau) d \tau
\end{aligned}
$$

with the kernels

$$
\begin{array}{cc}
K_{A}=-2 H_{1}-\frac{1}{2 \pi}, & L_{A}=-2 H_{2}, \\
K_{B}=-2 \kappa^{2} \beta H_{1}, & L_{B}=-2 \kappa^{2} \beta H_{2}+1
\end{array}
$$

where we set $\beta(t, \tau):=z^{\prime}(t) \cdot z^{\prime}(\tau)$ for $0 \leq t, \tau \leq 2 \pi$. These kernels are infinitely differentiable and satisfy

$$
K_{A}(t, t)=0, \quad 0 \leq t \leq 2 \pi
$$

For all $p \geq 0$, the infinite differentiability of the kernels and the property (1.13) implies that $A: H^{p}[0,2 \pi] \rightarrow H^{p+2}[0,2 \pi]$ is bounded [11, Theorem 13.20]), whereas, without the vanishing diagonal values 
for $K_{B}$, we only have that $B: H^{p}[0,2 \pi] \rightarrow H^{p+1}[0,2 \pi]$ is bounded [11, Theorem 12.15].

Now we can summarize our task into designing and analyzing a numerical method for the approximate numerical solution of a hypersingular integral equation of the form (1.10) with infinitely differentiable kernels $K_{A}, L_{A}, K_{B}$ and $L_{B}$ satisfying (1.13). We note that the kernels must not necessarily be of the form (1.12). Given the fact that, as a consequence of its positive eigenvalues according to (1.9), the operator $T_{0}$ is strictly coercive, a Galerkin method offers the advantage of a straightforward convergence analysis $[\mathbf{3}, \mathbf{6}, \mathbf{9}, \mathbf{1 3}]$. However, designing, analyzing and implementing a fully discrete variant is more involved than the corresponding task for a collocation method. Therefore the latter seems to be preferable.

The double-layer approach to the solution of the exterior Neumann problem suffers from non-uniqueness issues when $-\kappa^{2}$ is a Neumann eigenvalue for the Laplace operator in $\Omega$. Analogously, the singlelayer approach fails when $-\kappa^{2}$ is a Dirichlet eigenvalue. The most efficient remedy for this deficiency is a formulation by a combined single- and double-layer potential, for details see [7, 8]. For ease of presentation, here in our motivation for the proposed discretization of the normal derivative of the double-layer potential via Maue's formula and trigonometric differentiation, we have chosen only to present the pure double-layer potential. However, we note that, via changing the kernels of the operator $B$ and adding a multiple of the identity operator $I: H^{p}[0,2 \pi] \rightarrow H^{p}[0,2 \pi]$, the convergence analysis of the following sections also applies to the combined single- and double-layer approach.

For an alternative fully discrete method for the hypersingular equation (1.2) and, more generally, the combined single- and double-layer equation via regularization of the hypersingular operator and trigonometric collocation, including an error analysis in Sobolev spaces, we refer to a recent paper by Boubendir and Turc [2].

The plan for developing a collocation method in this paper is as follows. In Section 2 we will introduce a semi-discrete collocation method via trigonometric interpolation with an even number of equidistant collocation points and establish a convergence result in the Sobolev space setting. This is followed in Section 3 by a fully discrete variant where the integral operators $A$ and $B$ are approximated by quadrature op- 
erators $A_{n}$ and $B_{n}$ via trigonometric interpolation quadratures that take proper care of the logarithmic singularities of $A$ and $B$. The differentiation operator $D$ will be approximated by trigonometric differentiation, i.e., by differentiation of the trigonometric interpolation polynomial. Again, we will establish a convergence result for the fully discrete version and conclude with a numerical example exhibiting the rapid convergence for smooth data.

The collocation method as described in [10] also exploits Maue's formula. However, only for the inner derivative trigonometric differentiation is employed. The derivative of the single-layer potential is reduced to a Hilbert transform and this results in a more complicated operation for splitting off the logarithmic singularities. As a result, the method proposed in this paper is much easier to implement than the method in [10].

A similar method, without convergence analysis, has been recently employed by Cakoni and Kress [4] for a closely related hypersingular integral equation arising from the solution of a generalized impedance boundary value problem.

2. Semi-discrete collocation. We begin by describing a semidiscrete method by collocation via trigonometric interpolation. Let $X_{n}$ be the space of trigonometric polynomials of degree less than or equal to $n$ of the form

$$
\varphi(t)=\sum_{m=0}^{n} \alpha_{m} \cos m t+\sum_{m=1}^{n-1} \beta_{m} \sin m t
$$

and denote by $P_{n}$ the interpolation operator that maps $2 \pi$-periodic functions $g$ into the unique trigonometric polynomial $P_{n} g$ that interpolates $\left(P_{n} g\right)\left(t_{j}\right)=g\left(t_{j}\right)$ at the equidistant interpolation points $t_{j}:=\pi j / n$ for $j=0, \ldots, 2 n-1$. For the interpolation error, we note that

$$
\left\|P_{n} g-g\right\|_{q} \leq \frac{C}{n^{p-q}}\|g\|_{p}, \quad 0 \leq q \leq p, \frac{1}{2}<p,
$$

for all $g \in H^{p}[0,2 \pi]$ and some constant $C$ depending on $p$ and $q$ (see $[\mathbf{1 1}$, Theorem 11.8]).

From (1.9), we observe that $T_{0}$ maps $X_{n}$ into itself. Hence, we can use the set $t_{j}=j \pi / n, j=0, \ldots, 2 n-1$, as collocation points. We 
assume unique solvability of (1.10) and approximate the solution $\varphi$ by a trigonometric polynomial $\varphi_{n} \in X_{n}$ satisfying the projected equation

$$
T_{0} \varphi_{n}-P_{n} D A D \varphi_{n}-P_{n} B \varphi_{n}=P_{n} f .
$$

For this semi-discrete collocation method we can state the following result.

Theorem 2.1. Under the assumption that the operator $T_{0}-D A D-$ $B$ is bijective, the semi-discrete collocation method given by (2.3) converges in $H^{p}[0,2 \pi]$ for each $p \geq 1$.

Proof. All constants occurring in this proof depend on $p$. For $p \geq 1$, using (2.2) and the boundedness of $D: H^{p}[0,2 \pi] \rightarrow H^{p-1}[0,2 \pi]$ and $A: H^{p-1}[0,2 \pi] \rightarrow H^{p+1}[0,2 \pi]$, we obtain

$$
\left\|D P_{n} A D \varphi-D A D \varphi\right\|_{p-1} \leq \frac{c_{1}}{n}\|\varphi\|_{p}
$$

for all $\varphi \in H^{p}[0,2 \pi]$ and some constant $c_{1}$. With the triangle inequality, again using (2.2), we can estimate

$$
\begin{aligned}
\left\|P_{n} D \psi-D P_{n} \psi\right\|_{p-1} & \leq\left\|P_{n} D \psi-D \psi\right\|_{p-1}+\left\|D\left(\psi-P_{n} \psi\right)\right\|_{p-1} \\
& \leq \frac{c_{2}}{n}\|\psi\|_{p+1}
\end{aligned}
$$

for all $\psi \in H^{p+1}[0,2 \pi]$ and some constant $c_{2}$. From this, setting $\psi=A D \varphi$ and using the boundedness of $A: H^{p}[0,2 \pi] \rightarrow H^{p+2}[0,2 \pi]$, we find that

$$
\left\|P_{n} D A D \varphi-D P_{n} A D \varphi\right\|_{p-1} \leq \frac{c_{3}}{n}\|\varphi\|_{p}
$$

for all $\varphi \in H^{p}[0,2 \pi]$ and some constant $c_{3}$. Now we combine (2.4) and (2.5) to obtain

$$
\left\|P_{n} D A D \varphi-D A D \varphi\right\|_{p-1} \leq \frac{c_{4}}{n}\|\varphi\|_{p}
$$

for all $\varphi \in H^{p}[0,2 \pi]$ and some constant $c_{4}$.

Using (2.2) and the boundedness of $B: H^{p}[0,2 \pi] \rightarrow H^{p}[0,2 \pi]$, we can estimate

$$
\left\|P_{n} B \varphi-B \varphi\right\|_{p-1} \leq \frac{c_{5}}{n}\|\varphi\|_{p}
$$


for all $\varphi \in H^{p}[0,2 \pi]$ and some constant $c_{5}$. From this estimate and (2.6), it follows that $\left\|P_{n}(D A D+B)-(D A D+B)\right\|_{H^{p} \rightarrow H^{p-1}} \rightarrow 0$, and the assertion of the theorem follows from the standard convergence analysis for projection methods for operator equations that are compact perturbations of a principal operator that leaves the approximation space invariant (see [11, Theorem 13.12]).

3. Fully discrete collocation. We now proceed with describing a fully discrete method for which we have to approximate both the integral operators $A$ and $B$ and the differentiation operator $D$. To this end, for the latter, we give a short description of trigonometric differentiation which approximates $D$ by $D_{n}:=D P_{n}$, i.e., the derivative $D g$ of a $2 \pi$-periodic function $g$ by the derivative $D_{n} g$ of the trigonometric interpolation polynomial $P_{n} g \in X_{n}$. From the Lagrange basis

$L_{k}(t)=\frac{1}{n}\left\{1+2 \sum_{m=1}^{n-1} \cos m\left(t-t_{k}\right)+\cos n\left(t-t_{k}\right)\right\}, \quad k=0, \ldots, 2 n-1$,

for trigonometric interpolation by summation and straightforward differentiation we obtain that

$$
\left(D_{n} g\right)\left(t_{j}\right)=\sum_{k=0}^{2 n-1} d_{k-j}^{(n)} g\left(t_{k}\right), \quad j=0, \ldots, 2 n-1,
$$

where

$$
d_{j}^{(n)}=\left\{\begin{array}{cl}
\frac{(-1)^{j}}{2} \cot \frac{j \pi}{2 n}, & j= \pm 1, \ldots, \pm(2 n-1), \\
0, & j=0 .
\end{array}\right.
$$

From (2.2), we immediately have the error estimate

$$
\left\|D_{n} g-D g\right\|_{q-1} \leq \frac{C}{n^{p-q}}\|g\|_{p}, \quad 0 \leq q \leq p, \frac{1}{2}<p,
$$

for all $g \in H^{p}[0,2 \pi]$ and some constant $C$ depending on $p$ and $q$.

For the approximations $A_{n}$ and $B_{n}$, we use the quadrature operators 
defined via interpolatory quadratures

$$
\begin{aligned}
\left(A_{n} \varphi\right)(t):= & \frac{1}{2 \pi} \int_{0}^{2 \pi}\left\{\left[P_{n}\left(K_{A}(t, \cdot)\right) \varphi\right](\tau) \ln \left(4 \sin ^{2} \frac{t-\tau}{2}\right)\right. \\
& \left.+\left[P_{n}\left(L_{A}(t, \cdot)\right) \varphi\right](\tau)\right\} d \tau
\end{aligned}
$$

and the analogous expression for $B_{n}$. From (1.7) and the Lagrange basis (3.1), the discrete form

$$
\begin{aligned}
& \left(A_{n} \varphi\right)\left(t_{j}\right):=\sum_{k=0}^{2 n-1}\left\{R_{k-j}^{(n)} K_{A}\left(t_{j}, t_{k}\right)+\frac{1}{2 n} L_{A}\left(t_{j}, t_{k}\right)\right\} \varphi\left(t_{k}\right), \\
& j=0, \ldots, 2 n-1,
\end{aligned}
$$

of $A_{n}$ (and correspondingly of $B_{n}$ ) can be deduced with the weights

$$
R_{j}^{(n)}=-\frac{1}{n}\left\{\sum_{m=1}^{n-1} \frac{1}{m} \cos m t_{j}+\frac{1}{2 n} \cos n t_{j}\right\}, \quad j=0, \pm 1, \ldots, \pm(2 n-1),
$$

(see [11, Chapter 12]). For these approximations, the error estimates

$$
\left\|B_{n} \varphi-B \varphi\right\|_{q+1} \leq \frac{C}{n^{p-q}}\|\varphi\|_{p}, \quad 0 \leq q \leq p, \frac{1}{2}<p,
$$

for all $\varphi \in H^{p}[0,2 \pi]$ and some constant $C$ depending on $p$ and $q$ (and correspondingly for $A$ ) are available (see [11, Theorem 12.18]). Further, due to $K_{A}(t, t)=0$, the estimate on $A_{n}-A$ can be strengthened into

$$
\left\|\left(P_{n} A_{n}-P_{n} A\right) \varphi\right\|_{q+1} \leq \frac{C}{n^{p-q+1}}\|\varphi\|_{p}, \quad 0 \leq q \leq p, \frac{1}{2}<p,
$$

for all trigonometric polynomials $\varphi$ of degree less than or equal to $n$ and some constant $C$ depending on $p$ and $q$.

The latter estimates now can be used to prove convergence for the fully discrete method.

Theorem 3.1. Under the assumption that the operator $T_{0}-D A D-B$ is bijective, the fully discrete collocation method converges in $H^{p}[0,2 \pi]$ for each $p>3 / 2$. 
Proof. For all trigonometric polynomials $\varphi$ of the form (3.1), in view of $D_{n} \varphi=D \varphi$, we can transform

$$
\begin{aligned}
P_{n}\left(D_{n} A_{n} D_{n}-D A D\right) \varphi & =P_{n} D\left(P_{n} A_{n}-A\right) D \varphi \\
& =P_{n} D P_{n}\left(A_{n}-A\right) D \varphi+P_{n} D\left(P_{n} A-A\right) D \varphi .
\end{aligned}
$$

Note that the interpolation operators $P_{n}: H^{p}[0,2 \pi] \rightarrow H^{p}[0,2 \pi]$ are uniformly bounded for $p>1 / 2$ as consequence of (2.2). Then for $p>3 / 2$, with the aid of (3.6) for the first term on the right-hand side, we can estimate

$$
\left\|P_{n} D P_{n}\left(A_{n}-A\right) D \varphi\right\|_{p-1} \leq \frac{c_{1}}{n}\|\varphi\|_{p}
$$

for all trigonometric polynomials $\varphi$ of the form (3.1) and some constant $c_{1}$. For the second term, from (2.2) and the boundedness of $A$ : $H^{p-1}[0,2 \pi] \rightarrow H^{p+1}[0,2 \pi]$, we conclude that

$$
\left\|P_{n} D\left(P_{n} A-A\right) D \varphi\right\|_{p-1} \leq \frac{c_{2}}{n}\|\varphi\|_{p}
$$

for all trigonometric polynomials $\varphi$ of the form (3.1) and some constant $c_{2}$. Combining both estimates, we find that

$$
\left\|P_{n}\left(D_{n} A_{n} D_{n}-D A D\right) \varphi\right\|_{p-1} \leq \frac{c_{3}}{n}\|\varphi\|_{p}
$$

for all trigonometric polynomials $\varphi$ of the form (3.1) and some constant $c_{3}$. The boundedness of $P_{n}$ and the estimate (3.5) imply that

$$
\left\|P_{n}\left(B_{n}-B\right) \varphi\right\|_{p-1} \leq \frac{c_{4}}{n}\|\varphi\|_{p}
$$

for all $\varphi \in H^{p}[0,2 \pi]$ and some constant $c_{4}$. Combining (3.7) and (3.8), we arrive at the estimate

$$
\left\|P_{n}\left(D_{n} A_{n} D_{n}-D A D\right) \varphi\right\|+\left\|P_{n}\left(B_{n}-B\right)\right\|_{p-1} \leq \frac{c}{n}\|\varphi\|_{p}
$$

for all trigonometric polynomials $\varphi$ of the form (3.1) and some constant $c$.

From (3.3) and (3.5), applied to $A_{n}-A$, we conclude uniform boundedness of $D_{n}: H^{p}[0,2 \pi] \rightarrow H^{p-1}[0,2 \pi]$ and $A_{n}: H^{p-1}[0,2 \pi] \rightarrow$ $H^{p}[0,2 \pi]$. In view of the uniform boundedness of $P_{n}: H^{p}[0,2 \pi] \rightarrow$ $H^{p}[0,2 \pi]$, this implies that the operators

$$
P_{n}\left(D_{n} A_{n} D_{n}-D A D\right): H^{p}[0,2 \pi] \rightarrow H^{p-1}[0,2 \pi]
$$


are uniformly bounded. For a fixed trigonometric polynomial $\varphi$, we have $D_{n} \varphi=D \varphi$ for all sufficiently large $n$. For these $n$ we can write

$$
\left(D_{n} A_{n} D_{n}-D A D\right) \varphi=D_{n}\left(A_{n}-A\right) D \varphi+\left(D_{n}-D\right) A D \varphi,
$$

and consequently the estimates (3.3) and (3.5) imply that

$$
\left\|P_{n}\left(D_{n} A_{n} D_{n}-D A D\right) \varphi\right\|_{p-1} \longrightarrow 0, \quad n \rightarrow \infty,
$$

that is, we have pointwise convergence for all trigonometric polynomials. By the Banach-Steinhaus theorem, this implies pointwise convergence for all $\varphi \in H^{p}[0,2 \pi]$. Together with the estimate (3.5) for $B_{n}-B$, we now have pointwise convergence of the operators

$$
P_{n}\left(D_{n} A_{n} D_{n}+B_{n}-A-B\right): H^{p}[0,2 \pi] \rightarrow H^{p-1}[0,2 \pi]
$$

to zero. From this and the estimate (3.9), the statement of the theorem again follows by applying standard convergence results for collocation methods (see [11, Corollary 13.13]).

We note that, from the general error analysis of, for example, [11, Theorem 13.12 and Corollary 13.13] the approximation error is determined by the interpolation error for the exact solution and how well the quadrature operators $A_{n}$ and $B_{n}$ approximate $A$ and $B$ for the exact solution. Therefore, in particular, we have super algebraic convergence of order $O\left(n^{-m}\right)$ for all $m \in \mathbf{N}$ if the exact solution is infinitely differentiable.

From the Lagrange basis (3.1) and the eigenvalues (1.9) we obtain that

$$
\left(P_{n} T_{0} \varphi\right)\left(t_{j}\right)=\sum_{k=0}^{2 n-1} b_{k-j}^{(n)} \varphi\left(t_{k}\right), \quad j=0, \ldots, 2 n-1,
$$

for all trigonometric polynomials $\varphi$ of degree $n$ of the form (3.1) where $(3.10)$

$$
b_{j}^{(n)}=\left\{\begin{array}{clrl}
\frac{1}{2 n}+\frac{1}{4 n}\left[(-1)^{j}-1\right] \sin ^{-2} \frac{j \pi}{2 n}, & & j= \pm 1, \ldots, \pm(2 n-1), \\
\frac{n^{2}+1}{2 n}, & j=0 .
\end{array}\right.
$$

With this, our fully discrete collocation method leads to the linear system for the nodal values of the approximating trigonometric polynomial 
$\varphi^{(n)}$ in the form

$$
\sum_{k=0}^{2 n-1}\left(U_{j k}-V_{j k}\right) \varphi^{(n)}\left(t_{k}\right)=f\left(t_{j}\right), \quad j=0, \ldots, 2 n-1 .
$$

Here the matrix

$$
U_{j k}:=b_{k-j}^{(n)}-\sum_{\ell=0}^{2 n-1} \sum_{m=0}^{2 n-1} d_{\ell-j}^{(n)}\left\{R_{m-\ell}^{(n)} K_{A}\left(t_{\ell}, t_{m}\right)+\frac{1}{2 n} L_{A}\left(t_{\ell}, t_{m}\right)\right\} d_{k-m}^{(n)}
$$

corresponds to the operator $T_{0}-A$ and the matrix

$$
V_{j k}:=R_{k-j}^{(n)}\left[K_{B}\left(t_{j}, t_{k}\right)\right]+\frac{1}{2 n} L_{B}\left(t_{j}, t_{k}\right)
$$

corresponds to the operator $B$. Note that the two summations in the expression for $U_{j k}$ represent multiplications by Toeplitz matrices, i.e., convolutions. These can be efficiently implemented via fast Fourier transform techniques. With this in mind, we note that our proposed fully discrete collocation method, in principle, is of the same computational cost as the method from [10].

From the eigenvalues of the principal operator $T_{0}$ given by (1.9) we observe that the condition number of the matrix $U_{j k}-V_{j k}$ grows linearly with $n$. However, since in the case of infinitely differentiable kernels and right hand sides due to the super algebraic convergence, we need only a comparatively small $n$ to achieve an acceptable approximation error, it is numerically safe to apply our collocation method.

We explicitly note that the extra weights $b_{j}^{(n)}$ are required since the approximation of $T_{0}$ by $D_{n} S_{0} D_{n}$ is not exact for $c_{n}(t):=\cos n t$. On the one hand, we have $T_{0} c_{n}=n c_{n}$, but, on the other hand, $D_{n} S_{0} D_{n} c_{n}=0$. This effect would not occur if we would work with an odd number of interpolation and collocation points. However, this would be at the expense of making the implementation of a multi-grid method, for example, more involved.

Finally, we note that the mean value operator $M$ entered our analysis only to make the operator $T_{0}$ injective. In the implementation, obviously, we can enter the operator $M$ into the kernel $L_{B}$ and subtract $1 / 2 n$ from each of the weights $b_{j}^{(n)}$. 

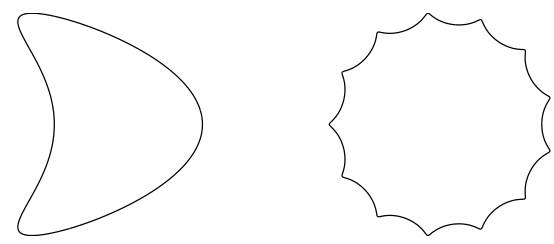

FiguRE 1. Kite-shaped and star-shaped domain for numerical examples.

TABLE 1. Numerical results for kite shape and plane wave.

\begin{tabular}{|c|r|c|c|r|c|}
\hline & $n$ & $\operatorname{Re} u_{\infty}(d)$ & $\operatorname{Im} u_{\infty}(d)$ & \multicolumn{1}{|c|}{$\operatorname{Re} u_{\infty}(-d)$} & $\operatorname{Im} u_{\infty}(-d)$ \\
\hline \multirow{3}{*}{$\kappa=1$} & 8 & -0.509409962488 & 1.102428968355 & -1.409345531762 & 0.142066539014 \\
& 16 & -0.509186826181 & 1.102342227578 & 1.409008701432 & 0.142326199772 \\
& 32 & -0.509187204400 & 1.102342295466 & -1.409008962514 & 0.142325857120 \\
& 64 & -0.509187204401 & 1.102342295465 & 1.409008962515 & 0.142325857119 \\
& & & & & \\
& 16 & -1.383456607313 & 2.001387579839 & 0.489336208993 & 0.398562627488 \\
& 32 & -1.275907059347 & 1.947492520029 & 0.450263294961 & 0.563405909690 \\
& 64 & -1.275907056954 & 1.947492511552 & 0.450263294720 & 0.563405908743 \\
& 128 & -1.275907056954 & 1.947492511552 & 0.450263294720 & 0.563405908743 \\
\hline
\end{tabular}

4. Numerical examples. For a first numerical example, we consider the scattering of a plane wave $u^{i}(x)=e^{i \kappa x \cdot d}$ with incident direction $d \in S^{1}$ by a sound-hard or non-conducting cylinder with a non-convex kite-shaped cross section with boundary $\partial \Omega$ illustrated on the left in Figure 1 and described by the parametric representation

$$
z(t)=(\cos t+0.65 \cos 2 t-0.65,1.5 \sin t), \quad 0 \leq t \leq 2 \pi .
$$

In this case we want to determine the scattered wave $u$ such that the total wave $u+u^{i}$ has vanishing normal derivative on $\partial \Omega$, that is, we have to solve an exterior Neumann problem with normal derivative $g=-\partial_{\nu} u^{i}$.

The coefficient $u_{\infty}$ in the asymptotics

$$
u(x)=\frac{e^{i \kappa|x|}}{\sqrt{|x|}}\left\{u_{\infty}\left(\frac{x}{|x|}\right)+O\left(\frac{1}{|x|}\right)\right\}, \quad|x| \rightarrow \infty,
$$

for the scattered wave $u$ is known as the far field pattern. From the 
asymptotics for the Hankel functions it can be deduced that the far field pattern of the double-layer potential (1.1) is given by

$$
u_{\infty}(\widehat{x})=\frac{e^{-i(\pi / 4)} \sqrt{\kappa}}{\sqrt{8 \pi}} \int_{\partial \Omega} \nu(y) \cdot \hat{x} e^{-i \kappa \widehat{x} \cdot y} \psi(y) d s(y), \quad \widehat{x} \in S^{1},
$$

which can be evaluated by the composite trapezoidal rule after solving the integral equation for $\psi$.

Table 1 gives some approximate values for the far field pattern $u_{\infty}(d)$ and $u_{\infty}(-d)$ in the forward direction $d$ and the backward direction $-d$. The direction $d$ of the incident wave is $d=(1,0)$. Note that the super algebraic convergence is clearly exhibited. Of course, the correct digits coincide with those obtained by the collocation method described in [10].

For a second example, we choose a star-shaped boundary $\partial \Omega$ illustrated on the right in Figure 1 and given by the parametric representation in complex notation

$$
z(t)=\exp \left(i t+\frac{c e^{i m t}}{4^{m}}-\frac{c}{e^{i m t}}\right), \quad 0 \leq t \leq 2 \pi,
$$

where $m=13$ and $c=0.7 / 13$. The incident direction $d$ is the same as in the first example. The numerical results are shown in Table 2. As is to be expected the more oscillatory boundary curve requires a higher discretization level $n$ to achieve the same accuracy as in the previous example.

Of course, from both examples we also observe that increasing the wave number requires a higher discretization level $n$ to maintain the same approximation accuracy. In general, any straightforward discretization of the boundary integral equations for boundary value problems for the Helmholtz equation works well only in the resonance region, that is, for wave numbers $\kappa$ for which the wave length $2 \pi / \kappa$ is smaller than or of the same magnitude as the diameter of the domain. For a survey on the adaption of the boundary integral equation approximations to high wave numbers we refer to Chandler-Wilde et al. [5].

We conclude with a numerical example for both domains with a known exact solution in order to make the convergence from the numerical tables more apparent. For this, we choose as exact solution 
TABLE 2. Numerical results for star shape and plane wave.

\begin{tabular}{|c|r|c|c|r|c|}
\hline & $n$ & $\operatorname{Re} u_{\infty}(d)$ & $\operatorname{Im} u_{\infty}(d)$ & \multicolumn{1}{|c|}{$\operatorname{Re} u_{\infty}(-d)$} & \multicolumn{1}{c|}{$\operatorname{Im} u_{\infty}(-d)$} \\
\hline \multirow{5}{*}{$\kappa=1$} & 16 & -0.041629668004 & 0.530214889497 & 0.514544507657 & 0.537833694958 \\
& 32 & -0.041875020775 & 0.529545444109 & -0.515861836232 & 0.537903598930 \\
& 64 & -0.041890426628 & 0.529469327428 & -0.515875310840 & 0.537788091372 \\
& 128 & -0.041890522430 & 0.529469261749 & -0.515875570355 & 0.537788529234 \\
& 256 & -0.041890522430 & 0.529469261749 & 0.515875570414 & 0.537788529192 \\
& & & & & \\
& 16 & -1.080763705679 & 1.445722775275 & 0.721225434505 & 0.752127533454 \\
& 32 & -0.753494745389 & 1.668418370719 & 0.824417998081 & 0.409260954432 \\
& 64 & -0.753496938314 & 1.666978089154 & -0.822969318435 & 0.409719468141 \\
& 128 & -0.753493509711 & 1.666978862290 & -0.822975646376 & 0.409722288223 \\
& 256 & -0.753493509977 & 1.666978862706 & -0.822975647420 & 0.409722286893 \\
\hline
\end{tabular}

TABLE 3. Numerical results for kite shape and point source.

\begin{tabular}{|c|r|c|c|c|c|}
\hline & $n$ & $\operatorname{Re} u_{\infty}(d)$ & $\operatorname{Im} u_{\infty}(d)$ & $\operatorname{Re} u_{\infty}(-d)$ & $\operatorname{Im} u_{\infty}(-d)$ \\
\hline \multirow{3}{*}{$\kappa=1$} & 8 & 0.998681880447 & 0.001542733424 & 1.000471734772 & 0.002049192877 \\
& 16 & 1.000000229594 & 0.000000930197 & 0.999999994442 & 0.000000088610 \\
& 64 & 1.000000000000 & 0.000000000000 & 1.000000000000 & 0.000000000000 \\
& & & & & \\
& & & & & \\
& 16 & 1.152668123650 & 0.064273945521 & 0.841261803679 & 0.025416899045 \\
& 32 & 1.000000009771 & 0.000000001597 & 1.000000003822 & 0.000000006164 \\
& 64 & 1.000000000000 & 0.000000000000 & 1.000000000000 & 0.000000000000 \\
\hline
\end{tabular}

TABLE 4. Numerical results for star shape and point source.

\begin{tabular}{|c|r|c|r|r|r|}
\hline & $n$ & $\operatorname{Re} u_{\infty}(d)$ & \multicolumn{1}{c|}{$\operatorname{Im} u_{\infty}(d)$} & \multicolumn{1}{c|}{$\operatorname{Re} u_{\infty}(-d)$} & \multicolumn{1}{c|}{$\operatorname{Im} u_{\infty}(-d)$} \\
\hline \multirow{3}{*}{$\kappa=1$} & 16 & 0.999792011079 & 0.000452444515 & 1.000002329811 & -0.000343406206 \\
& 32 & 1.000026351818 & -0.000086053085 & 0.999958434875 & 0.000084824252 \\
& 64 & 1.000000002022 & 0.000000000136 & 1.000000002022 & 0.000000000136 \\
& 128 & 1.000000000000 & 0.000000000000 & 1.000000000000 & 0.000000000000 \\
& & & & & \\
& 16 & 0.790790974460 & 0.184999288758 & 1.034796971567 & 0.166191310541 \\
& 32 & 1.000030820871 & 0.000217847783 & 0.999975406461 & 0.000095443329 \\
& 64 & 0.999999479597 & 0.000000185474 & 0.999999492323 & 0.000000154847 \\
& 128 & 1.000000000040 & 0.000000000059 & 0.999999999939 & 0.000000000067 \\
& 256 & 1.000000000000 & 0.000000000000 & 1.000000000000 & 0.000000000000 \\
\hline
\end{tabular}

a point source at the origin (contained in $\Omega$ ), i.e.,

$$
u(x)=\frac{1}{4} \sqrt{8 \pi \kappa} e^{i(\pi / 4)} H_{0}^{(1]}(\kappa|x|),
$$

which has constant far field pattern $u_{\infty}=1$. Tables 3 and 4 show the numerical results for the far field patterns in the direction $d$ and $-d$ for $d=(1,0)$. In summary, all four examples are in agreement with 
our theoretical results on the convergence of the proposed fully discrete collocation method.

Acknowledgments. Part of this research was carried out while the author was visiting the Institut Mittag-Leffler, Djursholm, in spring 2013 during the scientific program on Inverse Problems and Applications. The hospitality and the support are gratefully acknowledged.

\section{REFERENCES}

1. K.E. Atkinson, The numerical solution of integral equations of the second kind, Cambridge University Press, Cambridge 1997.

2. Y. Boubendir and C. Turc, Wave-number estimates for regularized combined field boundary integral operators in acoustic scattering problems with Neumann boundary conditions, IMA J. Numer. Anal. 33 (2013), 1176-1225.

3. T. Cai, A fast solver for a hypersingular boundary integral equation, Appl. Numer. Math. 59 (2009), 1960-1969.

4. F. Cakoni and R. Kress, Integral equation methods for the inverse obstacle problem with generalized impedance boundary condition, Inv. Prob. 29 (2013), 015005 .

5. S.N. Chandler-Wilde, I.G. Graham, S. Langdon and E.A. Spence, Numericalasymptotic boundary integral methods in high-frequency acoustic scattering, Acta Numer. 21 (2012), 89-305.

6. D. Chien and K.E. Atkinson, A discrete Galerkin method for a hypersingular boundary integral equation, IMA J. Numer. Anal. 17 (1997), 453-478.

7. D. Colton and R. Kress, Integral equation methods in scattering theory, SIAM, Philadelphia, 2013.

8. _ Inverse acoustic and electromagnetic scattering theory, 3rd ed., Springer, New York, 2013.

9. R. Kieser, B. Kleemann and A. Rathsfeld, On a full discretization scheme for a hypersingular boundary integral equation over smooth curves, Z. Anal. Anwend. 11 (1992), 385-396.

10. R. Kress, On the numerical solution of a hypersingular integral equation in scattering theory, J. Comp. Appl. Math. 61 (1995), 345-360.

11. L Linear integral equations, 3rd ed., Springer, New York, 2014.

12. A.W. Maue, Über die Formulierung eines allgemeinen Beugungsproblems durch eine Integralgleichung, Z. Physik 126 (1949), 601-618.

13. W. McLean and O. Steinbach, Boundary element preconditioners for a hypersingular integral equation on an interval, Adv. Comp. Math. 11 (1999), 271278.

University of Göttingen, Lotzestrasse 16-18 D-37083 Göttingen, Germany Email address: kress@math.uni-goettingen.de 